\title{
Novel microfilaricidal activity of nanosilver
}

This article was published in the following Dove Press journal:

International Journal of Nanomedicine

21 February 2012

Number of times this article has been viewed

\section{Sunil K Singh' \\ Kalyan Goswami² \\ Richa D Sharma² \\ Maryada VR Reddy ${ }^{2}$ \\ Debabrata Dash' \\ 'Department of Biochemistry, Institute of Medical Sciences, Banaras Hindu University, Varanasi, ${ }^{2}$ Department of Biochemistry, Mahatma Gandhi Institute of Medical Sciences, Sevagram, India}

Correspondence: Debabrata Dash Department of Biochemistry, Institute of Medical Sciences, Banaras Hindu University, Varanasi 221005, India

$\mathrm{Tel}+9|93369| 0665$

Fax +9I 5422367568

Email ddass@satyam.net.in
Purpose: The currently available drug repertoire against lymphatic filariasis, a major health hazard in the developing world, is inadequate and is fraught with serious limitations. Thus, the development of an effective antifilarial strategy has become a global research thrust mandated by the World Health Organization. Nanoparticles of silver endowed with antibacterial potency are known to induce apoptosis in eukaryotic cells. The present study was designed to investigate the possible microfilaricidal efficacy of silver nanoparticles and to establish the validity of apoptotic rationale in antifilarial drug designing.

Methods: This report analyzed the effect of nanoparticles of silver as well as gold (size range: $10-15 \mathrm{~nm}$ ) on the microfilariae of Brugia malayi obtained from the lavage of peritoneal cavities of infected jirds (Meriones unguiculatus). The study included a microfilarial motility assay, a trypan blue exclusion test, a poly(adenosine diphosphate-ribose) polymerase activity study, ethidium bromide/acridine orange differential staining, and transmission, as well as scanning electron microscopic evaluation of ultrastructural changes in microfilariae.

Results: The study demonstrates that nanoparticles of silver, but not of gold, elicited significant loss in microfilarial motility. Differential staining of parasites with ethidium bromide and acridine orange, poly(adenosine diphosphate-ribose) polymerase activity in microfilarial lysate, and electron microscopic findings underscored apoptotic death of parasites attributable to nanosilver. In a trypan blue exclusion test, the $50 \%$ lethal dose of nanosilver was measured to be $101.2 \mu \mathrm{M}$, which was higher than the recorded complete inhibitory concentration value $(50.6 \mu \mathrm{M})$, thus supporting nanosilver as a potential drug candidate against lymphatic filariasis.

Conclusion: The present report provides the first ever conclusive proof in support of apoptosis as a novel stratagem in antifilarial drug designing and nanoscale silver as a valid lead in research on antifilarial therapeutics. The main embargo about the current drug diethylcarbamazine citrate is its empirical use without rationale. Effective microfilaricidal activity of nanosilver at relatively low concentrations as reported in this study, with evidence of the induction of apoptosis in microfilariae, projects nanosilver as a potential drug adjuvant against lymphatic filariasis. The much higher $50 \%$ lethal dose value of nanosilver compared to the complete inhibitory concentration value reported in this study argues in favor of a safe therapeutic window of this agent in its antifilarial efficacy

Keywords: silver nanoparticles, apoptosis, lymphatic filariasis, microfilaricidal agent, parasitic diseases

\section{Introduction}

Human lymphatic filariasis is a major vector-borne disease in countries of tropical and subtropical regions caused by the nematode parasites Wuchereria bancrofti and 
Brugia malayi. The adult forms of the parasites harbor in host lymphatic tissue, whereas the microfilarial forms (Mf) circulate in the blood as a reproductive product. The latter are transmitted to the mosquito vector where the larval (infective) stage is generated. Passage of infective larvae into humans and subsequent development into adult worms complete the life cycle. The disease has varied presentations depending on the host immune status. Despite accelerated research to develop an effective therapeutic regimen against the disease, the antifilarial drug inventory still remains limited to diethylcarbamazine citrate (DEC). ${ }^{1}$ Since last century, DEC has been almost the sole antifilarial drug, and its inherent disadvantages such as unwanted side effects, lack of patient compliance, and poor macrofilaricidal effectiveness ${ }^{2}$ have warranted research on new antifilarial drug development. The World Health Organization has placed special emphasis on the development of novel drugs against human lymphatic filariasis, realizing the severe socioeconomic and emotional burden of this disease on developing nations. ${ }^{3}$ An estimated populace of approximately 120 million clinical cases of lymphatic filariasis, with another 751 million people living in endemic areas, are bare statistics of this harsh fact. ${ }^{4}$ According to the Indian Council of Medical Research, more than 550 million people are exposed to filarial infection in India, which is estimated to be $40 \%$ of the total global burden of the disease. ${ }^{5}$

The natural response of the host to the microbial pathogen is inflammation. ${ }^{6}$ Oxidative stress is a major contributing factor to innate immunity, ${ }^{7}$ and hence generation of a pro-oxidant state provides a premise for antifilarial drug development. The authors have earlier reported potential antifilarial properties of traditional therapeutic herbal extracts composed of polyphenolics ${ }^{8}$ and synthetic inhibitors of dihydrofolate reductase. ${ }^{9}$ Nanoparticles of silver (AgNPs) endowed with antibacterial potency ${ }^{10,11}$ induced p53 expression and apoptosis in eukaryotic cells. ${ }^{12,13}$ Strikingly, free radical generation has been implicated in the apoptosis induced by AgNPs. ${ }^{14-16}$ DEC was also shown to induce microfilarial apoptosis. ${ }^{17} \mathrm{DEC}$ acts in an innate response-mimetic manner by recruitment of macrophages and by invoking proinflammatory status. ${ }^{6}$ The importance of apoptosis in the host-parasite relationship is not itself a unique concept; ${ }^{18}$ however, it has never been explored as an antifilarial therapeutic strategy. With this perspective, the present study was designed to investigate the possible microfilaricidal efficacy of AgNPs and to establish the validity of apoptotic rationale in antifilarial drug designing.

\section{Materials and methods}

\section{Synthesis and characterization of biocompatible nanoparticles}

AgNPs were synthesized following the procedure described in earlier reports. ${ }^{11,19,20}$ In short, $0.017 \mathrm{~g}$ silver nitrate was dissolved in deionized water along with sodium hydroxide $(0.01 \mathrm{M})$ and liquid ammonia $(2 \%)$ to form a $0.01 \mathrm{M}$ solution of stable soluble complex of silver ions. D-glucose and hydrazine (each at $0.01 \mathrm{M}$ concentration) were added to the solution of silver ions to ensure its complete reduction at a final concentration of $0.005 \mathrm{M}$. The $\mathrm{pH}$ of the solution was adjusted to 7.4 with citric acid. The final solutions were carefully stored in glass vials at $4^{\circ} \mathrm{C}$ for further characterization. The size, morphology, and distribution of AgNPs were characterized using a transmission electron microscope (Tecnai 12; Philips, Eindhoven, the Netherlands) and an ultraviolet-visible spectrophotometer (GE Life Sciences, Uppsala, Sweden). Nanoparticles were found to be spherical in shape with average size of 10-15 nm (Figure 1). Before each experiment, the solution containing the nanoparticles was sonicated (Labsonic ${ }^{\circledR}$ 2000; B Braun Melsungen AG, Melsungen, Germany) for about 2 minutes and passed through filters of $0.2 \mu \mathrm{m}$ pore size (Sartorius Stedim Biotech, Gottingen, Germany). Gold nanoparticles (AuNPs) (G1527; $10 \mathrm{~nm}$ diameter) were procured from Sigma-Aldrich Corporation (Bangalore, India).

\section{Collection and preparation of $B$. malayi microfilariae}

Microfilariae of $B$. malayi were obtained by lavage from the peritoneal cavities of infected jirds (Meriones unguiculatus). The Mf used for in vitro experiments were collected, washed with Roswell Park Memorial Institute (RPMI) 1640 medium supplemented with antibiotics (gentamicin $20 \mathrm{mg}$; penicillin $100 \mathrm{mg}$; streptomycin $100 \mathrm{mg}$ ), $15 \mathrm{mM}$ 4-(2-hydroxyethyl)1-piperazineethanesulfonic acid, organic acids (malic acid, $\alpha$-ketoglutaric acid, D-succinic acid, and fumaric acid at concentrations of $670,370,60$, and $55 \mathrm{mg} / \mathrm{L}$, respectively) and sugars (sucrose and fructose at concentrations of $26.7 \mathrm{~g} / \mathrm{L}$ and $0.4 \mathrm{~g} / \mathrm{L}$, respectively). The use of animals for the study was approved by the Institutional Animal Ethics Committee which follows the Committee for the Purpose of Control and Supervision of Experiments on Animals norms.

\section{In vitro screening for antifilarial activity against B. malayi}

In vitro screening for antifilarial activity against $B$. malayi was carried out as described earlier. ${ }^{8}$ Approximately $100 \mathrm{Mf}$ 
A

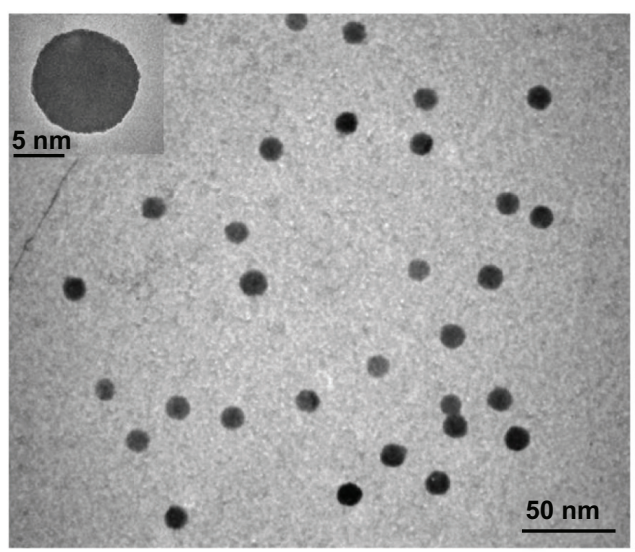

B

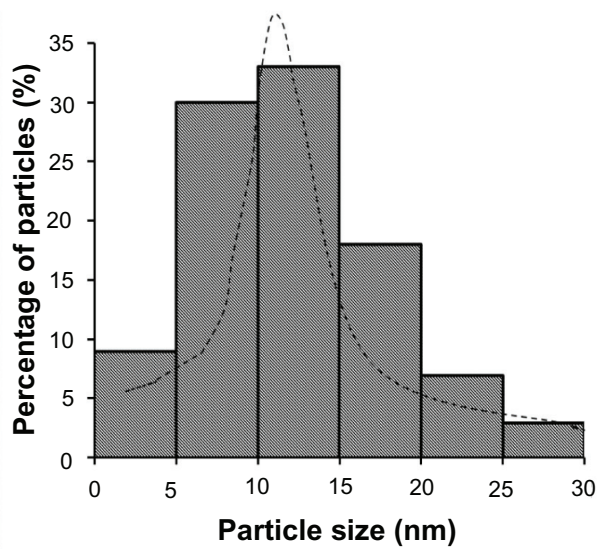

D

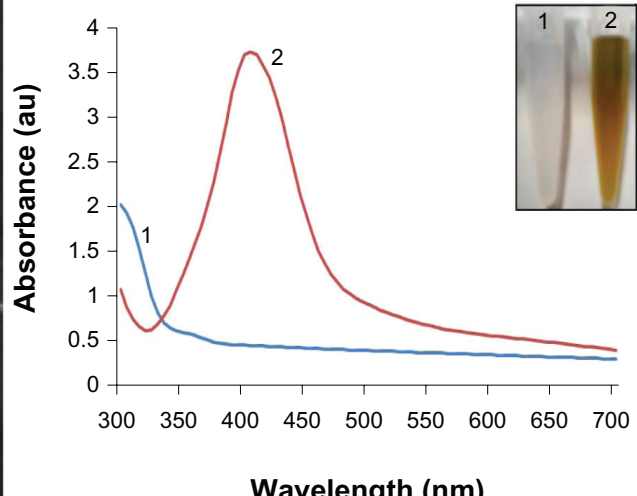

Wavelength (nm)

C

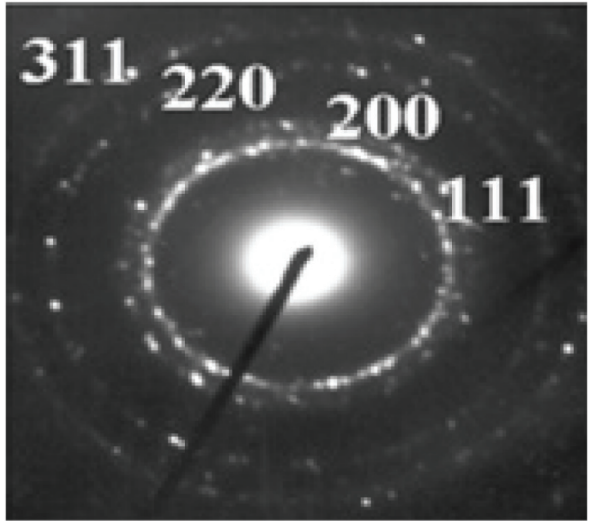

Figure I Characterization of silver nanoparticles. (A) Silver nanoparticles showing spherical, monodispersed particles (scale bar, $50 \mathrm{~nm}$ ). The inset shows one single particle of silver (scale bar, $5 \mathrm{~nm}$ ). (B) Particle size distribution showing preponderance of particles in the size range of $10-15 \mathrm{~nm}$. (C) Electron diffraction pattern of nanoparticles showing various crystallographic planes. (D) Optical spectra of silver before (I) and after reduction (2). The inset shows the corresponding change in color.

(in $100 \mu \mathrm{L} \mathrm{RPMI)} \mathrm{were} \mathrm{introduced} \mathrm{into} \mathrm{each} \mathrm{well} \mathrm{of} \mathrm{24-well}$ microculture plates. B. malayi microfilariae were screened for the antifilarial effect of AgNPs in vitro over a wide dose range $(1-100 \mu \mathrm{M})$. Microfilariae were treated similarly with AuNPs of comparable sizes to rule out any nonspecific nanoparticle effect. As a positive control, staurosporine - a known inducer of apoptosis - was added to the microfilariae at $0.5 \mu \mathrm{M}$ (elicits $100 \%$ loss in motility), whereas Mf in RPMI medium, in the absence of any agent added, was used as a negative control (vehicle). The plates were incubated for 48 hours at $37^{\circ} \mathrm{C}$ in the presence of $5 \%$ carbon dioxide. Subsequently, the number of live and dead Mf in each well was counted under an inverted microscope (Nikon Corporation, Tokyo, Japan) and the percentage of motile Mf out of total Mf recruited per aliquot was calculated.

\section{Determination of $50 \%$ lethal dose for nanosilver}

Cytotoxicity of AgNPs was evaluated by a trypan blue dye exclusion assay. Peripheral blood mononuclear cells $\left(1 \times 10^{5}\right.$ cells $\left./ \mathrm{mL}\right)$ were exposed to varying concentrations of AgNPs for 48 hours followed by incubation with trypan blue $(0.2 \mathrm{mg} / \mathrm{mL})$ for 1 minute. Cells were observed under a Nikon light microscope (Tokyo, Japan) and the viable cell ratio were calculated by counting the stained and unstained cells separately. ${ }^{21}$ Viable cells do not uptake trypan blue, whereas nonviable cells with porous membranes stain blue. The cytotoxicity of the nanoparticles was evaluated and the $50 \%$ lethal dose was determined.

\section{Poly(adenosine diphosphate-ribose) polymerase (PARP) activity assay}

PARP activity in B. malayi microfilariae was determined using a commercial kit ( R \& D Systems Inc, Minneapolis, MD) according to the manufacturer's instruction. Briefly, $100 \mu \mathrm{L}$ aliquots of suspension (containing about $100 \mathrm{Mf}$ ) were treated with different reagents and lysed with $1 \%$ Triton X-100 (Himedia laboratories Pvt Ltd, Mumbai, India) in the presence of protease inhibitors. Lysate $(20 \mu \mathrm{g})$ was added to each well in 96-well plates precoated with histone. 
PARP activity was determined from the incorporation of biotinylated poly(adenosine diphosphate-ribose) onto immobilized histone, which was measured by the addition of steptavidin-conjugated horseradish peroxidise and a suitable chromogenic substrate to the incubation mixture. A standard curve for PARP enzymatic activity $\left(\mathrm{A}_{450}\right.$ versus PARP units) was initially generated using $0.01,0.05,0.1,0.5$, and 1 unit of enzyme per well. The absorbance obtained with each test sample (microfilarial lysate) was extrapolated on the standard curve to obtain the corresponding PARP activity. The control sample (microfilaria without any pretreatment) provided $100 \%$ activity reference point. The percentage inhibition in enzymatic activity in other test samples (lysates treated with different reagents) was accordingly calculated. The experiment was carried out in triplicate and the percentage inhibition was averaged over the experiments.

\section{Ethidium bromide/Acridine orange $(E B / A O)$ staining for the detection of apoptosis}

Dual staining with $\mathrm{EB} / \mathrm{AO}$ was carried out as described elsewhere. $^{22}$ The dye mix consisted of $100 \mu \mathrm{g} / \mathrm{mL} \mathrm{EB}$ and $100 \mu \mathrm{g} / \mathrm{mL}$ AO in phosphate-buffered saline. Microfilariae (control as well as treated with different reagents for 48 hours) were washed and resuspended in $25 \mu \mathrm{L}$ cold phosphate-buffered saline, followed by the addition of $5 \mu \mathrm{L}$ EB/AO dye mix. Stained microfilariae were viewed under an epifluorescence microscope (Nikon) with the excitation filter set at 480/30 nm and the barrier filter at 535/40 nm. Tests were carried out in triplicate, counting a minimum of $10 \mathrm{Mf}$ in each observation.

\section{Electron microscopy}

Mf were treated with staurosporine, AgNPs, or AuNPs for 48 hours. Samples were fixed in Karnovsky fixative ( $\mathrm{pH} 7.2$ ) for 2 hours at $4^{\circ} \mathrm{C}$ followed by postfixation in osmium tetroxide (1\%) and then dehydrated in ascending concentrations of acetone. For scanning electron microscopy, dehydrated samples were critical point dried, mounted on an aluminum stub with adhesive tape, and sputter-coated with colloidal gold. Specimens were viewed under a Leo 435VP scanning electron microscope (LEO Electron Microscopy Ltd, Cambridge, UK) at an operating voltage of $15 \mathrm{kV}$. For transmission electron microscopy, blocks were prepared as previously described. ${ }^{23}$ Ultrathin sections (60-70 nm thick) were prepared with an ultramicrotome (Leica EM UC6; Leica Microsystems GmbH, Wetzlar, Germany). Sections were contrasted with uranyl acetate and alkaline lead citrate. Specimens were mounted on formvar-coated grids and viewed under a FEI Morgagni ${ }^{\mathrm{TM}}$ 268(D) (FEI, Hillsboro, OR) digital transmission electron microscope at $120 \mathrm{kV}$ using image analysis software from Soft Imaging System GmbH (Muenster, Germany). The final magnifications were derived from the photo micrographs and the scale bars determined.

\section{Results and discussion}

AgNPs were synthesized through the aqueous chemical precipitation method, as described earlier. ${ }^{11,19,20}$ Nanoparticles were spherical in shape, 10-15 nm in diameter (Figure 1A), and monodispersed with a narrow particle size distribution (Figure 1B). The selected area electron diffraction pattern from these particles matched the crystallographic planes of the face-centered cubic AgNPs (Figure 1C). Ultravioletvisible absorption spectra showed the reduction of silver ions into the AgNPs under ambient conditions (Figure 1D). The inset shows the color changes before and after the process of reduction. The silver nitrate solution exhibited maximum absorbance at $300 \mathrm{~nm}$, which gradually underwent red shift with the appearance of a sharp peak at $410 \mathrm{~nm}$, which can be attributed to a narrow size distribution of the particles formed in the solution.

The microfilariae remained viable and motile in RPMI media, as expected. The effect of AgNPs (10-15 nm) upon Mf motility was evaluated over a wide concentration range. Remarkably, at $4.6 \mu \mathrm{M}$, the AgNPs evoked motility loss in $50 \%$ of Mf population, whereas at $50.6 \mu \mathrm{M}$, the AgNPs rendered the entire Mf population immotile (Table 1). The AuNPs $(10-15 \mathrm{~nm})$ failed to produce any significant antifilarial effect at the identical concentrations. However, staurosporine $(0.5 \mu \mathrm{M})$ as a positive control induced complete loss in Mf motility.

Table I Brugia malayi microfilariae were incubated with silver nanoparticles at varying concentrations

\begin{tabular}{lc}
\hline $\begin{array}{l}\text { Concentration } \\
\text { of AgNPs }(\mu \mathrm{M})\end{array}$ & $\begin{array}{l}\text { Percentage loss } \\
\text { in Mf motility }\end{array}$ \\
\hline 4.6 & $50.0 \pm 2.63$ \\
9.2 & $76.5 \pm 0.88$ \\
13.8 & $85 \pm 1.17$ \\
18.4 & $89 \pm 0$ \\
23 & $92 \pm 0.58$ \\
27.6 & $94 \pm 0.58$ \\
32.2 & $98 \pm 0$ \\
36.8 & $97.5 \pm 0.29$ \\
41.4 & $99 \pm 0$ \\
50.6 & $100 \pm 0$ \\
\hline
\end{tabular}

Notes: The percentage loss in the motility of microfilarial forms was recorded after 48 hours. Each experiment was carried out in triplicate and the results were presented as mean \pm standard error of the mean.

Abbreviations: AgNPs, silver nanoparticles; Mf, microfilarial forms. 
When evaluated for the extent of cytotoxicity by trypan blue exclusion test, AgNPs at $101.2 \mu \mathrm{M}$ elicited 50\% lethality.

The inhibition of PARP activity is a valid measure for the assessment of cellular apoptosis. ${ }^{24}$ During apoptosis, PARP is cleaved by caspase 3 with an ensuing reduction in PARP enzyme activity, which thus prevents apoptotic cells from repairing their own DNA. ${ }^{25}$ PARP activity in Mf exposed to nanosilver $(50 \mu \mathrm{M})$, nanogold $(50 \mu \mathrm{M})$, or staurosporine $(0.5 \mu \mathrm{M})$ was then studied. Both AgNPs and staurosporine induced nearly $70 \%$ attenuation in PARP enzymatic activity, whereas only $25 \%$ loss in activity was observed in the presence of AuNPs (Table 2), thus consistent with the significant microfilaricidal effect of nanoscale silver.

The interaction between microfilariae and nanosilver was further investigated with $\mathrm{EB}$ and $\mathrm{AO}$ differential staining. ${ }^{22}$ AO permeates into healthy live cells to stain the nuclear material green, whereas cellular access of EB is restricted, unless there is membrane damage. Mf treated with AgNPs $(50 \mu \mathrm{M})$ and staurosporine $(0.5 \mu \mathrm{M})$ were stained orange-yellow with EB (Figure 2B and C), which reflected loss in surface membrane integrity. This contrasted with the profile of control parasites as well as that of Mf exposed to AuNPs $(50 \mu \mathrm{M})$, which were stained green with AO (Figure 2A and D).

Nanosilver-induced ultrastructural changes in microfilariae were subsequently investigated by electron microscopy. Scanning electron microscopy images reflected marked loss in microfilarial sheath in parasites treated either with staurosporine $(0.5 \mu \mathrm{M})$ or AgNPs $(50 \mu \mathrm{M})$, whereas RPMI-treated control microfilariae or parasites exposed to AuNPs $(50 \mu \mathrm{M})$ exhibited clear undamaged translucent sheaths (Figure 3).

Transmission electron microscopy images of ultrathin sections of microfilariae, pretreated either with staurosporine or AgNPs, revealed multiple large, round vacuoles inside cells (Figure 4). On the contrary, vacuoles were scanty in parasites treated with AuNPs and absent in RPMI-treated control parasites, which possessed thick homogeneous cuticles

Table 2 Percentage inhibition in poly(adenosine diphosphateribose) polymerase activity in the microfilariae treated with different reagents as indicated compared with control (Roswell Park Memorial Institute medium only)

\begin{tabular}{ll}
\hline Reagents & $\begin{array}{l}\text { Percentage inhibition } \\
\text { in PARP activity } \\
\text { (mean } \pm \text { SEM) }\end{array}$ \\
\hline AgNPs $(50 \mu \mathrm{M})$ & $71.62 \pm 3.23$ \\
AuNPs $(50 \mu \mathrm{M})$ & $25.88 \pm 1.09$ \\
Staurosporine $(0.5 \mu \mathrm{M})$ & $67.82 \pm 4.13$ \\
\hline
\end{tabular}

Abbreviations: AgNPs, silver nanoparticles; AuNPs, gold nanoparticles; PARP poly(adenosine diphosphate-ribose) polymerase; SEM, standard error of the mean.

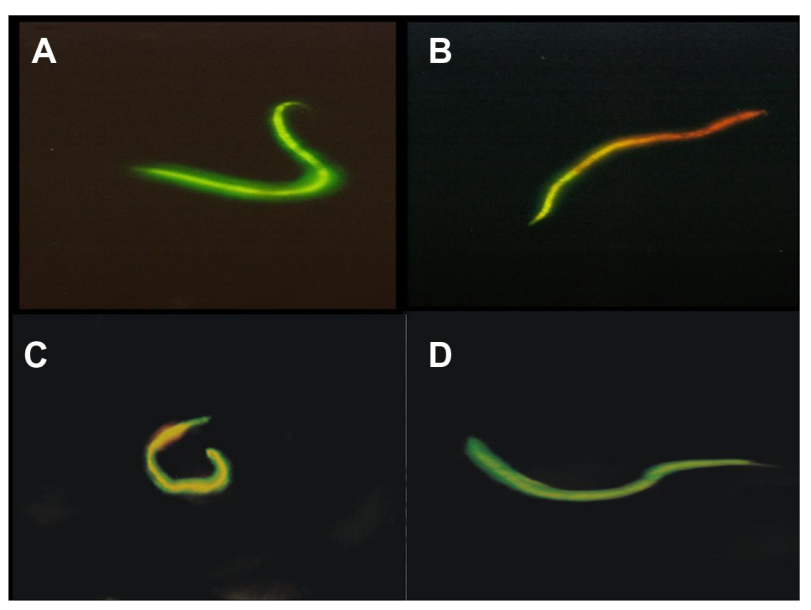

Figure 2 Ethidium bromide/acridine orange differential staining of microfilarial forms for the detection of apoptosis. Untreated $(\mathbf{A})$ and gold nanoparticles preincubated (D) nuclei showed green staining due to acridine orange permeation, while organisms treated with silver nanoparticles (B) and staurosporine (C) appeared orange-yellow due to ethidium bromide, suggesting loss of integrity of surface membrane of the parasite shown.

Note: Data are representative of three different experiments.

and intact cell organelles without vacuoles. The presence of vacuoles in ultrastructural sections of staurosporine-treated as well as AgNP-treated microfilariae further confirmed the apoptotic rationale in the pharmacodynamics of AgNPs. It may be noted that, conventional mechanisms for the study of apoptosis such as terminal deoxynucleotidyl transferase-mediated deoxyuridine triphosphate nick end labeling assay and DNA fragmentation are not technically viable in a parasite model. ${ }^{17}$ Taken together, the above findings were consistent with apoptotic changes induced in microfilariae in the presence of nanosilver similar to that elicited by staurosporine. Nanogold of comparable size and concentration, on the other hand, had no adverse effect on parasites.

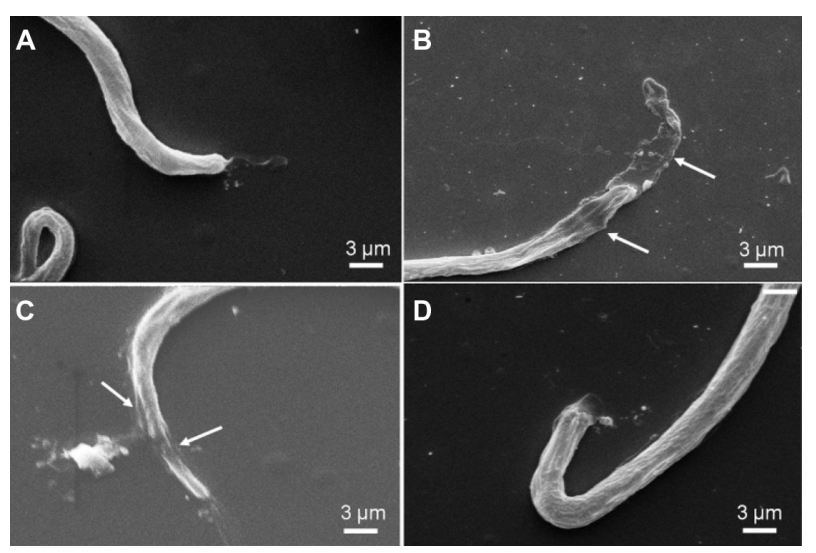

Figure 3 Scanning electron micrographs of (A) untreated control parasite in Roswell Park Memorial Institute medium; (B) microfilariae treated with staurosporine $(0.5 \mu \mathrm{M})$; (C) microfilariae treated with silver nanoparticles $(50 \mu \mathrm{M})$, and $(D)$ microfilariae treated with gold nanoparticles $(50 \mu \mathrm{M})$. Note: Data are representative of three different experiments. 


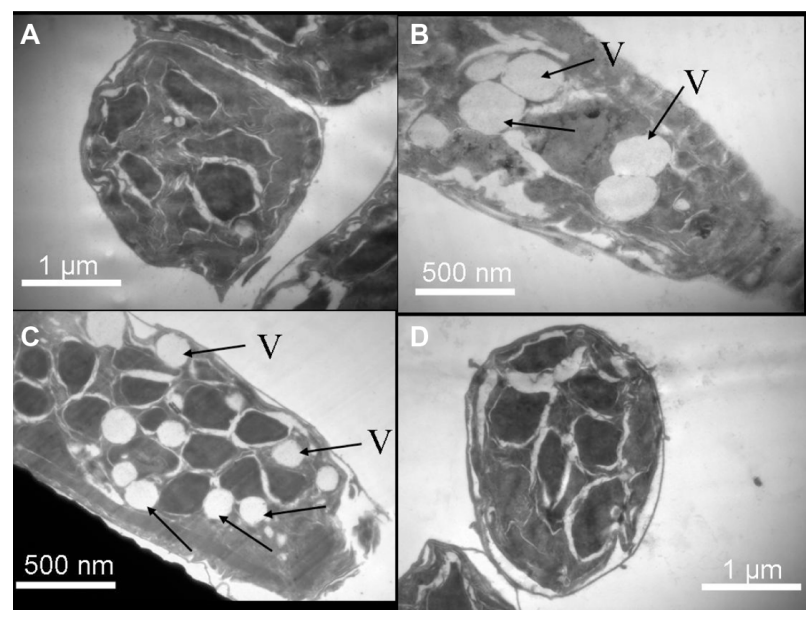

Figure 4 Transmission electron micrographs through sections of (A) untreated control parasite in Roswell Park Memorial Institute medium; (B) microfilariae treated with staurosporine $(0.5 \mu \mathrm{M})$; (C) microfilariae treated with silver nanoparticles $(50 \mu \mathrm{M})$; and (D) microfilariae treated with gold nanoparticles $(50 \mu \mathrm{M})$.

Notes: Arrows point to the vacuoles; data are representative of three different experiments.

Abbreviation: $\mathrm{V}$, vacuoles.

DEC has been shown to induce apoptosis in the microfilariae of $W$. bancrofti,${ }^{17}$ though the drug is not known to be filaricidal in vitro. ${ }^{26}$ Thus, apoptosis induction in vitro by DEC may not be sufficient to kill the parasite, which could couple with the innate inflammatory response of the host to elicit effective antifilarial activity in vivo. In support of this, the cuticles of adult and microfilarial B. malayi were found to be resistant to hydrogen peroxide, a strong oxidant as well as apoptosis-inducing agent, which is attributable to the presence of $\alpha$-tocopherol in the lipid fraction of the parasite surface..$^{27}$ Interestingly, DEC in combination with hydrogen peroxide was found to have marked synergism against filarial parasites. ${ }^{28}$ The above facts underpinned the relative ineffectiveness of DEC as the sole filaricidal agent and prompts researchers to strategize combinations with apoptosis inducers as a novel lead for antifilarial therapeutics.

In an earlier report, the enhanced antibacterial potency of AgNPs was analyzed. ${ }^{11}$ Commendable efforts have been made to explore the underlying molecular mechanism of the antimicrobial activity of silver. ${ }^{29}$ Dissolved silver ions could be responsible for some of the biological actions of AgNPs against microorganisms $\mathrm{s}^{30,31}$ through their interaction with and inhibition of the thiol groups of vital enzymes. ${ }^{29,32}$ It has been recently demonstrated that the rate of ionization of silver from nanoparticles is significantly affected by the presence of salts and biomolecules in the suspension medium. ${ }^{33}$ Biological media contain large numbers of interfering molecules in abundance, which can thus influence the release of silver ions. It is unlikely that silver ions will rise to a significant level under the present experimental conditions, as reducing agents like glucose present in RPMI would keep these ions in the reduced state, and high concentration of chloride and phosphate ions in the medium would generate insoluble salts of silver (eg, silver chloride and silver phosphate). Besides this, the presence of citric acid during the process of AgNP synthesis would result in surface coating of the nanoparticles with citrate ions which would further limit the release of silver ions, as reported recently. ${ }^{33}$ Thus, the observed antimicrofilarial activity reported in this study is most likely attributable to AgNPs per se and not to silver ions.

\section{Conclusion}

The main embargo on the current drug DEC is its empirical use without rationale. DEC-based mass drug administration policy demands longer durations of therapy, which may lack patient compliance and thus lead to frequent failure. ${ }^{34} \mathrm{AgNPs}$, on the other hand, are capable of inducing apoptosis in mammalian cells. ${ }^{14-16}$ The effective microfilaricidal activity of AgNPs at relatively low concentration as reported in this study, with evidence of apoptotic induction in microfilariae, can project nanosilver as a potential drug adjuvant against lymphatic filariasis. Unlike DEC, which is effective against microfilariae only in vivo and not in vitro, ${ }^{35}$ nanosilver promises greater therapeutic effectiveness. Remarkably, a recent report has demonstrated the therapeutic potential of AgNPs against leishmaniasis, another parasitic disease caused by Leishmania tropica. ${ }^{36}$ However, the antiparasitic mechanism elucidated in this study was oxidative attack by AgNPs, which was further potentiated by the concomitant ultraviolet exposure. This mechanism is hardly of therapeutic relevance in the present study as filarial parasites are known to be endowed with robust antioxidant defenses. On the contrary, based on evidence presented in the present study, apoptosis is proposed as the mechanistic basis of the antifilarial activity of nanosilver. Interestingly, there is a subtle link reported between oxidative stress and apoptosis induction. ${ }^{37}$ Filarial parasites are lymphatic system-specific organisms. Given the wide distribution attainable by AgNPs in tissues including lymphatics, ${ }^{38}$ administered nanosilver is expected to achieve a desired local antifilarial therapeutic concentration in the infected population. Nanosilver may act synergistically with DEC and thus may be effective either individually or as an adjunct (at lower individual doses) to this standard drug, after critical evaluation of safety parameters. Although there have been concerns regarding the toxicity of nanoparticles in general, ${ }^{39}$ and 
silver in particular, ${ }^{16,40}$ nanosilver might be considered to have a safe therapeutic window. The $50 \%$ lethal dose of nanosilver $(100 \mu \mathrm{M})$, which is double the complete inhibitory concentration value $(50 \mu \mathrm{M})$ as reported in the present study, supports this contention. The report on Leishmania parasites has demonstrated the synergism between nanosilver and ultraviolet exposure in their antiparasitic effect. ${ }^{36}$ This study did not find any serious toxicity concern with silver. Thus, it can be inferred that AgNPs may be used as an adjuvant to DEC to potentiate synergistic apoptotic impact, so that the dose requirement of nanosilver may be further scaled down. To summarize, the present report provides the first ever conclusive proof in support of apoptosis as a novel stratagem in antifilarial drug designing and nanosilver as a valid lead in the research on antifilarial therapeutics.

\section{Acknowledgments}

Grants received by D Dash from the Indian Council of Medical Research and the Department of Biotechnology (DBT), Government of India, and the equipment support from the Department of Science and Technology Unit on Nanoscience and Technology, Banaras Hindu University, are gratefully acknowledged. K Goswami, RD Sharma, and MVR Reddy would like to thank DBT for funding the "Repository for Filarial Parasite and Reagents.” Electron microscopy was carried out at the Sophisticated Analytical Instrument Facility (Department of Science and Technology), All India Institute of Medical Sciences, New Delhi, India. SK Singh is a recipient of a research fellowship from the University Grants Commission, New Delhi, India.

\section{Disclosure}

The authors report no conflicts of interest in this work.

\section{References}

1. Ottesen EA, Duke BO, Karam M, Behbehani K. Strategies and tools for the control/elimination of lymphatic filariasis. Bull World Health Organ. 1997;75(6):491-503.

2. Fan PC. Diethylcarbamazine treatment of bancroftian and malayan filariasis with emphasis on side effects. Ann Trop Med Parasitol. 1992;86(4):399-405.

3. Tropical Diseases, Special Programme for Research and Training (TDR). Strategic direction for lymphatic filariasis research: disease burden and epidemiological trends [article on the Internet]. Geneva: TDR; 2012. Available from: http://www.who.int/tdr/diseases/lymphfil/direction/en/ index.html. Accessed February 3, 2012.

4. World Health Organization. Defining the roles of vector control and xenomonitoring in the Global Programme to Eliminate Lymphatic Filariasis. Report of the informal consultation; January 29-31, 2002; Geneva, Switzerland. Geneva: World Health Organization; 2002. Document: WHO/CDS/CPE/PVC/2002.3.

5. Indian Council of Medical Research. Prospects of elimination of lymphatic filariasis in India. ICMR Bull. 2002;32(5-6):1-14.
6. McGarry HF, Plant LD, Taylor MJ. Diethylcarbamazine activity against Brugia malayi microfilariae is dependent on inducible nitricoxide synthase and the cyclooxygenase pathway. Filaria J. 2005; 4:4-12.

7. Verhasselt V, Goldman M, Willems F. Oxidative stress up-regulates IL-8 and TNF-alpha synthesis by human dendritic cells. Eur J Immunol. 1998;28(11):3886-3890.

8. Sahare KN, Anandharaman V, Meshram VG, et al. Anti-microfilarial activity of methanolic extract of Vitex negundo and Aegle marmelos and their phytochemical analysis. Indian J Exp Biol. 2008;46(2): 128-131.

9. Bag S, Tawari NR, Sharma R, Goswami K, Reddy MV, Degani MS In vitro biological evaluation of biguanides and dihydrotriazines against Brugia malayi and folate reversal studies. Acta Trop. 2010;113(1): 48-51.

10. Kim JS, Kuk E, Yu KN, et al. Antimicrobial effects of silver nanoparticles. Nanomedicine. 2007;3(1):95-101.

11. Shrivastava S, Bera T, Roy A, Singh G, Ramchandrarao P, Dash D. Characterization of enhanced antibacterial effect of novel silver nanoparticles. Nanotechnology. 2007;18(22):225103-225111.

12. Gopinath P, Gogoi SK, Chattopadhyay A, Ghosh SS. Implications of silver nanoparticle induced cell apoptosis for in vitro gene therapy. Nanotechnology. 2008;19(7):075104-075113.

13. Gopinath P, Gogoi SK, Sanpui P, Paul A, Chattopadhyay A, Ghosh SS. Signaling gene cascade in silver nanoparticle induced apoptosis. Colloids Surf B Biointerfaces. 2010;77(2):240-245.

14. Foldbjerga R, Olesen P, Hougaard M, Dang DA, Hoffmann HJ, Autrup H. PVP-coated silver nanoparticles and silver ions induce reactive oxygen species, apoptosis and necrosis in THP-1 monocytes. Toxicol Lett. 2009;190(2):156-162.

15. Miura N, Shinohara Y. Cytotoxic effect and apoptosis induction by silver nanoparticles in HeLa cells. Biochem Biophys Res Commun. 2009;390(3):733-737.

16. AshaRani PV, Low Kuh Mun G, Hande MP, Valiyaveettil S. Cytotoxicity and genotoxicity of silver nanoparticles in human cells. ACS Nano. 2009;3(2):279-290.

17. Peixoto CA, Santos AC, Ayres CF. Molecular evidence for apoptosis in microfilariae of Wuchereria bancrofti induced by diethylcarbamazine. Parasitol Res. 2008;103(3):717-721.

18. Barcinski MA, DosReis GA. Apoptosis in parasites and parasite-induced apoptosis in the host immune system: a new approach to parasitic diseases. Braz J Med Biol Res. 1999;32(4):395-401.

19. Shrivastava S, Bera T, Singh SK, Singh G, Ramachandrarao P, Dash D. Characterization of antiplatelet properties of silver nanoparticles. ACS Nano. 2009;3(6):1357-1364.

20. Shrivastava S, Singh SK, Mukhopadhyay A, Sinha AS, Mandal RK, Dash D. Negative regulation of fibrin polymerization and clot formation by nanoparticles of silver. Colloids Surf B Biointerfaces. 2011;82(1):241-246.

21. Okuya S, Tanabe K, Tanizawa Y, Oka Y. Leptin increases the viability of isolated rat pancreatic islets by suppressing apoptosis. Endocrinology. 2001;142(11):4827-4830.

22. Ribble D, Goldstein NB, Norris DA, Shellman YG. A simple technique for quantifying apoptosis in 96-well plates. BMC Biotechnol. 2005;5:12-19.

23. Alves LC, Brayner FA, Silva LF, Peixoto CA. The ultrastructure of infective larvae (L3) of Wuchereria bancrofti after treatment with diethylcarbamazine. Micron. 2005;36(1):67-72.

24. Mullen P. PARP cleavage as a means of assessing apoptosis. In: Langdon SP, editor. Cancer Cell Culture: Methods and Protocols. Totowa, NJ: Humana Press; 2004:171-181.

25. Böhm I. The apoptosis marker enzyme poly-(ADP-ribose) polymerase (PARP) in systemic lupus erythematosus. Z Rheumatol. 2006;65(6): 541-544. German.

26. Mackenzie CD, Kron MA. Diethylcarbamazine: a review of its action in onchocerciasis, lymphatic filariasis and inflammation. Trop Dis Bull. 1985;82:R1-R36. 
27. Smith VP, Selkirk ME, Gounaris K. Brugia malayi: resistance of cuticular lipids to oxidant-induced damage and detection of $\alpha$-tocopherol in the neutral lipid fraction. Exp Parasitol. 1998;88(2):103-110.

28. Sharma RD, Janardhana PB, Gajalakshmi D, Reddy MV, Goswami K. Novel pharmaceutical rationale against human lymphatic filariasis: an oxidative premise. Asian Pac J Trop Med. 2009;2(1):30-34.

29. Morones JR, Elechiguerra JL, Camacho A, et al. The bactericidal effect of silver nanoparticles. Nanotechnology. 2005;16(10):2346-2353.

30. Hwang ET, Lee JH, Chae YJ, et al. Analysis of the toxic mode of action of silver nanoparticles using stress-specific bioluminescent bacteria. Small. 2008;4(6):746-750.

31. Choi O, Deng KK, Kim NJ, Ross L Jr, Surampalli RY, Hu Z. The inhibitory effects of silver nanoparticles, silver ions, and silver chloride colloids on microbial growth. Water Res. 2008;42(12):3066-3074.

32. Matsumura Y, Yoshikata K, Kunisaki S, Tsuchido T. Mode of bactericidal action of silver zeolite and its comparison with that of silver nitrate. Appl Environ Microbiol. 2003;69(7):4278-4281.

33. Kittler S, Greulich C, Diendorf J, Koller M, Epple M. Toxicity of silver nanoparticles increases during storage because of slow dissolution under release of silver ions. Chem Mater. 2010;22(16):4548-4554.
34. Nandha B, Sadanandane C, Jambulingam P, Das P. Delivery strategy of mass annual single dose DEC administration to eliminate lymphatic filariasis in the urban areas of Pondicherry, South India: 5 years of experience. Filaria J. 2007;6:7-12.

35. Maizels RM, Denham DA. Diethylcarbamazine (DEC): immunopharmacological interactions of an antifilarial drug. Parasitology. 1992; 105 Suppl:S49-S60.

36. Allahverdiyev AM, Abamor ES, Bagirova M, et al. Antileishmanial effect of silver nanoparticles and their enhanced antiparasitic activity under ultraviolet light. Int J Nanomedicine. 2011;6:2705-2714.

37. Buttke TM, Sandstrom PA. Oxidative stress as a mediator for apoptosis. Immunol Today. 1994;15(1):7-10.

38. Lankveld DP, Oomen AG, Krystek P, et al. The kinetics of the tissue distribution of silver nanoparticles of different sizes. Biomaterials. 2010;31(32):8350-8361.

39. El-Ansary A, Al-Daihan S. On the toxicity of therapeutically used nanoparticles: an overview. J Toxicol. 2009;2009:754810-754818.

40. El Badawy AM, Silva RG, Morris B, Scheckel KG, Suidan MT, Tolaymat TM. Surface charge-dependent toxicity of silver nanoparticles. Environ Sci Technol. 2011;45(1):283-287.
International Journal of Nanomedicine

\section{Publish your work in this journal}

The International Journal of Nanomedicine is an international, peerreviewed journal focusing on the application of nanotechnology in diagnostics, therapeutics, and drug delivery systems throughout the biomedical field. This journal is indexed on PubMed Central, MedLine, CAS, SciSearch $\AA$, Current Contents ${ }^{\circledR} /$ Clinical Medicine,

\section{Dovepress}

Journal Citation Reports/Science Edition, EMBase, Scopus and the Elsevier Bibliographic databases. The manuscript management system is completely online and includes a very quick and fair peer-review system, which is all easy to use. Visit http://www.dovepress.com/ testimonials.php to read real quotes from published authors. 